OPEN ACCESS

Edited by:

Giovanna D'Angelo,

University of Messina, Italy

Reviewed by:

Farhad Sattari,

University of Mohaghegh Ardabili, Iran

Giuseppe Cassone,

National Research Council (CNR), Italy

*Correspondence:

Mojtaba Madadi AsI

m.madadi@iasbs.ac.ir

Specialty section: This article was submitted to Condensed Matter Physics,

a section of the journal

Frontiers in Physics

Received: 18 June 2021 Accepted: 06 August 2021 Published: 19 August 2021

Citation:

Ramezani Akbarabadi S and Madadi Asl M (2021) Anchoring Groups Determine Conductance, Thermopower and Thermoelectric

Figure of Merit of an Organic Molecular Junction.

Front. Phys. 9:727325.

doi: 10.3389/fphy.2021.727325

\section{Anchoring Groups Determine Conductance, Thermopower and Thermoelectric Figure of Merit of an Organic Molecular Junction}

\author{
Saeideh Ramezani Akbarabadi ${ }^{1}$ and Mojtaba Madadi As/ ${ }^{2 *}$ \\ ${ }^{1}$ Department of Physics, University of Guilan, Rasht, Iran, ${ }^{2}$ Department of Physics, Institute for Advanced Studies in Basic \\ Sciences (IASBS), Zanjan, Iran
}

Transport properties of molecular junctions are prone to chemical or conformational modifications. Perturbation of the molecule-electrode coupling with anchoring groups or functionalization of the molecule with side groups is a well-characterized method to modulate the thermoelectric properties of molecular junctions. In this study, we used wideband approximation combined with the non-equilibrium Green's function (NEGF) formalism to inspect conductance, thermopower and figure of merit of an anthracene molecule coupled to gold (Au) electrodes. To provide a comparative study, three different anchoring groups were used, i.e., thiol, isocyanide and cyanide. The molecule was then perturbed with the amine side group in two positions to explore the interplay between anchoring groups and the side group. We showed that the introduction of side group alters transmission probability near the Fermi energy where transmission peaks are shifted relative to the Fermi level compared to the unperturbed molecule (i.e., without side group), ultimately leading to modified electrical and thermoelectric properties. The greatest value of electrical conductance was achieved when the side-group-perturbed molecule was anchored with isocyanide, whereas the thiol-terminated molecule perturbed with the side group yielded the greatest value of thermal conductance. We found that the Wiedemann-Franz law is violated in the Au-anthracene-Au device. Furthermore, the highest thermopower and figure of merit were attained in the cyanide-terminated perturbed molecule. Our results indicate that charge donating/accepting character of the anchoring group and its interplay with the side group position can modify temperature dependency of conductance, thermopower and figure of merit which is in agreement with experimental findings in organic molecular junctions. Such modifications may potentially contribute to the understanding of emerging conductance-based memory devices designed to mimic the behavior of brain-like synapses.

Keywords: anchoring group, side group, conductance, thermoelectric properties, anthracene molecule 


\section{INTRODUCTION}

Nanoscale thermoelectric devices where molecules are attached to two metal electrodes can be supposedly used for cooling, heating and power generation to address future energy concerns [1, 2]. An emerging application is low-dimensional solid-state heat pumps operating based on the thermoelectric effect which can be used to analyze temperature-dependent temporal dynamics of brain regions in animals [3, 4]. However, the efficiency of a thermoelectric device - termed as the figure of merit $(Z T)$ - poses practical limitation on the performance of the system since the figure of merit is determined by the interplay between several variables (see Eq. 10 below), i.e., thermopower or Seebeck coefficient $(S)$, electrical conductance $(G)$, thermal conductance $(\kappa)$ and temperature $(T)$. In this way, obtaining a high value for the thermoelectric figure of merit is challenging and requires novel materials with engineered physical and chemical properties [5-7].

In recent years, experimental techniques such as mechanically controllable break junction (MCBJ) $[8,9]$ and scanning tunneling microscope break junction (STM-BJ) [10-12] offered novel platforms to measure the thermoelectric properties at the level of molecules. A number of experiments employed STM-BJ based methods to carry out thermopower measurement in molecular junctions where a molecule is sandwiched between two metallic electrodes $[11,12]$. In this way, experimental studies were able to measure the thermopower of molecular structures with an interest in gold $(\mathrm{Au})$ electrodes [11, 12]. Later, Widawsky et al. used a STM-BJ based technique to simultaneously estimate the conductance and thermopower of molecular devices with $\mathrm{Au}$ electrodes obtained by direct measurement of electrical and thermoelectric currents [10]. Most recently, Mosso et al. employed a modified break junction technique in an Aulinked organic molecular junction, and for the first time, measured the electrical conductance and thermal conductance at the same time [13].

Several parameters can modify the thermoelectric properties of molecular junctions. Previously, several studies explored the effect of structural or chemical modification on the thermoelectric properties of molecular junctions by manipulating their transport properties. For example, it has been shown that the length of the molecule [14-17], functionalization of the molecule with side groups [18-21], intervention of anchoring groups [22-24], molecule-electrode coupling geometry $[25,26]$ and impurity doping $[7,27]$ can significantly tune the conductance, thermopower and figure of merit of molecular junctions, depending on the system. In this context, a key factor in the regulation of transport properties of metal-molecule-metal structures is the energy level alignment between Frontier molecular orbitals (FMOs) and the Fermi level of electrodes $[28,29]$ that can be modulated, e.g., by the geometric position or charge transfer nature of anchoring groups [24, 30, $31]$ or side groups [20, 21].

In fact, a suitable choice of the anchoring groups can favorably tune charge transport polarization through the molecular junction, i.e., p-type vs n-type [22, 31, 32]. More specifically, it has been shown that anchoring groups can theoretically determine the electrical conductance [22, 24, 33] and the thermal conductance [34] in molecular junctions. Such a modification occurs due to the realignment of energy levels relative to the Fermi energy of the electrodes, e.g., by chemical engineering of the anchoring units. For instance, thiol, isocyanide and cyanide are examples of widely used anchoring groups that attach to Au electrodes with different binding strengths and can dramatically disturb the symmetry of the transmission spectrum due to their charge-donating/ accepting character. In this way, the conductance of the molecular structure can be increased or decreased depending on the system [22-24]. In addition, the sign of the thermopower is determined by the anchoring group that reveals the nature of the transport through the modification of energy separation between the highest occupied molecular orbital (HOMO) and the lowest unoccupied molecular orbital (LUMO), i.e., the HOMO-LUMO gap [22, 30]. Experimental measurements done with STM-BJ [35] or MCBJ [22] techniques in molecular junctions with $\mathrm{Au}$ electrodes verified these theoretical predictions.

Perturbation of molecular systems with side groups in various positions can similarly modify the thermoelectric properties of the system [18-21]. The chemical character or the position of side groups can be a determinant of charge polarization between molecule and the electrodes due to the realignment of the FMOs relative to the Fermi energy of the electrodes [21, 36, 37]. Side group charge transfer nature (e.g., electron-donating amine unit vs electron-accepting nitro unit) can induce different resonance effects near the Fermi energy which shapes current flow in the system $[18,20]$. The emergence of side-group-induced Fano resonances can suppress thermal conductance in polycyclic organic molecules attached to Au electrodes, e.g., in the presence of the amine side group [37]. In the thiol-terminated molecules, the amine side group can attain the greatest figure of merit which is suppressed by changing the side group position [21]. In the isocyanide-terminated molecules, the methyl side group can yield the greatest thermoelectric figure of merit where its magnitude is relatively robust to the change of side group position [21]. Generally, these theoretical results were in agreement with experiments realized, e.g., by the STM-BJ techniques. For instance, it has been suggested that the electrical conductance is crucially determined by the side group contact geometry during mechanical modulation [38, 39].

The impact of anchoring groups or side groups on the conductance, thermopower and thermoelectric figure of merit of molecular junctions was taken into account separately in most of the previous studies. In this study, however, we assumed various settings to explore the interplay between anchoring groups and the side group position. We assumed an organic molecule where three different anchoring groups-thiol $(-\mathrm{SH})$, isocyanide $(-\mathrm{NC})$ and cyanide $(-\mathrm{CN})$ - mediated the coupling of the molecule to Au electrodes and then perturbed the molecule with the amine $\left(-\mathrm{NH}_{2}\right)$ side group in two different positions (comprising six distinct configurations). We explored the conductance, thermopower and thermoelectric figure of merit of the system using wide-band (WB) approximation combined with the non-equilibrium Green's function (NEGF) 


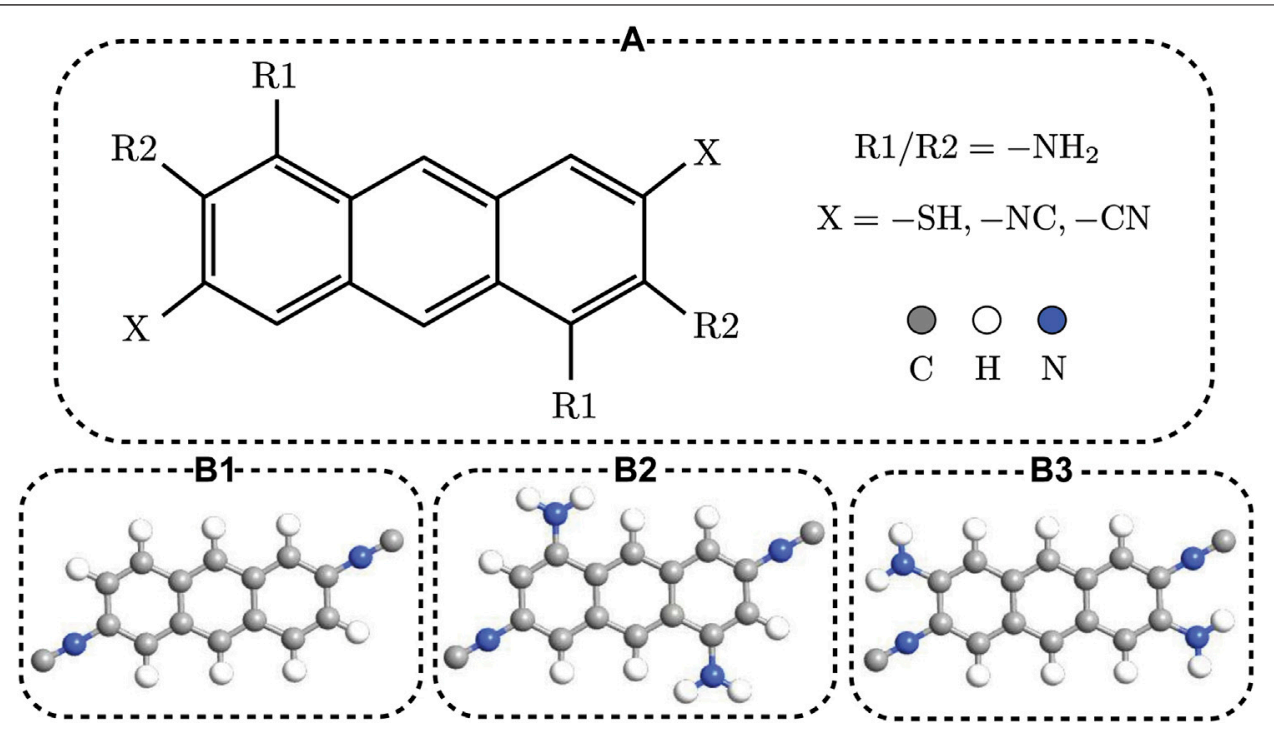

FIGURE 1 | (A) Schematic representation of the anthracene molecule terminated with anchoring groups $\mathrm{X}=$ thiol (-SH), isocyanide (-NC) or cyanide (-CN), and functionalized with the amine $\left(-\mathrm{NH}_{2}\right)$ side group in the $\mathrm{R} 1 / \mathrm{R} 2$ position. (B1-B3) Examplary model of the unperturbed isocyanide-terminated anthracene molecule (B1), and the same molecule perturbed with the amine side group in R1 (B2) and R2 (B3) positions.

formalism in the linear response regime. We considered anthracene $\left(\mathrm{C}_{14} \mathrm{H}_{10}\right)$ as the organic molecule that is a polycyclic aromatic molecule comprising three fused benzene rings. $\pi$-orbitals contributing in the transport make anthracene an appropriate choice for thermoelectric effect studies due to great conductance at the metal-molecule interface when the electrode is made of $\mathrm{Au}$ [40].

At first, we only considered the anchoring groups (i.e., unperturbed molecule) and observed the transmission spectrum and thermoelectric coefficients. We then perturbed the molecule with the amine side group (i.e., perturbed molecule) in two different positions, changed the anchoring groups, and made a comparative evaluation of the behavior of electrical conductance, thermal conductance, thermopower and figure of merit in various configurations. Our results indicate that in the molecule perturbed with the side group the transmission peaks (with adjusted probability) are displaced with respect to the Fermi energy compared to the unperturbed molecule. The gating of the transmission coefficient by anchoring groups or side groups can ultimately determine conductance, thermopower and thermoelectric figure of merit of the system. Furthermore, by calculating both the electrical conductance and electronic thermal conductance we found that the Wiedemann-Franz law is violated in the $\mathrm{Au}$-anthracene-Au molecular junction due to non-smooth dependence of the transmission coefficient on the energy near the Fermi level.

Finally, to present a comparison, the electrical conductance, electronic thermal conductance, thermopower and figure of merit at the room temperature were summarized for all configurations. The isocyanide-terminated molecule perturbed with the side group in the R1 position reached the highest electrical conductance, whereas the greatest value of the thermal conductance was ascribed to the thiol-terminated molecule perturbed with the side group in the R2 position. Notably, the R2-position perturbed molecule anchored by cyanide reached the higher thermopower and figure of merit at the room temperature with $Z T=0.25$. Our results show that the complex interaction between the chemical nature of anchoring groups and the position of the side group that perturbed the molecule is crucial for the determination of thermoelectric properties of the system where an appropriate combination of functionals can favorably enhance the thermoelectric efficiency.

\section{METHODS}

The anthracene molecule is schematically shown in Figure 1A. We assumed thiol $(-\mathrm{SH})$, isocyanide $(-\mathrm{NC})$ and cyanide $(-\mathrm{CN})$ as three different anchoring groups in the $\mathrm{X}$ position to mediate the coupling of the molecule to the Au electrodes. Furthermore, we used amine $\left(-\mathrm{NH}_{2}\right)$ side group in two different positions labeled as R1 and R2 in Figure 1A. One set of the molecular structures considered in this study is examplary shown in Figures 1B1-B3 comprising the unperturbed isocyanide-terminated anthracene molecule (B1), and the same molecule perturbed with the amine side group in R1 (B2) and R2 (B3) positions. The anthracene molecule was coupled to two three-dimensional $\mathrm{Au}$ electrodes mediated by each anchoring group. Thiol anchoring group was connected to the hollow position, whereas isocyanide and cyanide anchoring groups were connected to the top position of the Au (111) electrodes. $2 \times$ $2 \times 100 \mathrm{k}$-point sampling was used to sample the Brillouin zone. Then, the molecule was extended to include surface layers of the $\mathrm{Au}$ electrodes (three Au layers).

The Hamiltonian of the molecule $\left(H_{\mathrm{M}}\right)$ is calculated based on the extended Hückel method [41]: 


$$
H_{\mathrm{M}}=\frac{C}{2} S_{i j}\left(H_{i i}+H_{j j}\right),
$$

where $C=1.75$ is the Hückel coefficient, $S_{i j}$ indicates the overlap integral of the molecular orbitals, and $H_{i i}$ and $H_{j j}$ are the ionization energies of the orbitals. The transmission coefficient is calculated by the Landauer-Büttiker formalism expressed in terms of the Green's function [42]:

$$
T(\varepsilon)=\operatorname{Tr}\left[\mathbf{G}^{\mathrm{r}}(\varepsilon) \Gamma_{\mathrm{L}}(\varepsilon) \mathbf{G}^{\mathrm{a}}(\varepsilon) \Gamma_{\mathrm{R}}(\varepsilon)\right],
$$

where $\Gamma_{\mathrm{L} / \mathrm{R}}=-2 \operatorname{Im} \Sigma_{\mathrm{L} / \mathrm{R}}$ is given by the self-energy $\Sigma_{\mathrm{L} / \mathrm{R}}$ of the left (L) or right $(\mathrm{R})$ electrode. $\mathbf{G}^{\mathrm{r}}(\varepsilon)$ is the retarded Green's function of the molecule connected to the Au electrodes which in the NEGF formalism can be expressed as follows:

$$
\mathbf{G}^{\mathrm{r}}(\varepsilon)=\left(\varepsilon \mathbf{I}-H_{\mathrm{M}}-\Sigma_{\mathrm{L}}-\Sigma_{\mathrm{R}}\right)^{-1},
$$

where the advanced Green's function can be obtained by $\mathbf{G}^{\mathrm{a}}(\varepsilon)=$ $\left[\mathbf{G}^{\mathrm{r}}(\varepsilon)\right]^{\dagger}$.

Charge current $(I)$ and heat current $\left(I_{\mathrm{Q}}\right)$ are calculated based on the Landauer-Büttiker formalism [42]:

$$
\begin{aligned}
& I=\frac{e}{h} \int T(\varepsilon)\left[f_{\mathrm{L}}(\varepsilon)-f_{\mathrm{R}}(\varepsilon)\right] d \varepsilon, \\
& I_{\mathrm{Q}}=\frac{e}{h} \int(\varepsilon-\mu) T(\varepsilon)\left[f_{\mathrm{L}}(\varepsilon)-f_{\mathrm{R}}(\varepsilon)\right] d \varepsilon,
\end{aligned}
$$

where $e=1.6 \times 10^{-19} \mathrm{C}$ is the charge of electron, and $h=6.626 \times$ $10^{-34} \mathrm{~m}^{2} \mathrm{~kg} / \mathrm{s}$ is the Planck constant. $f_{\mathrm{L} / \mathrm{R}}(\varepsilon)$ is the Fermi distribution function of the left $(\mathrm{L})$ or right $(\mathrm{R})$ electrode. The Fermi distribution function is given by:

$$
f_{\mathrm{L} / \mathrm{R}}(\varepsilon)=\left[\exp \left(\left(\varepsilon-\mu_{\mathrm{L} / \mathrm{R}}\right) / k_{\mathrm{B}} T\right)+1\right]^{-1},
$$

where $k_{\mathrm{B}}=1.38 \times 10^{-23} \mathrm{JK}^{-1}$ is the Boltzmann constant.

The temperature gradient $(\Delta T)$ and voltage difference $(\Delta V)$ are negligible in the linear response regime, therefore, the flow of charge and heat currents in the system can be approximated in terms of $\Delta T$ and $\Delta V$ (to the first order) $[42,43]$ :

$$
\begin{aligned}
& I=e^{2} L_{0} \Delta V+\frac{e}{T} L_{1} \Delta T, \\
& I_{\mathrm{Q}}=-e L_{1} \Delta V-\frac{1}{T} L_{2} \Delta T,
\end{aligned}
$$

where $L_{n}=\hbar^{-1} \int d \varepsilon(\varepsilon-\mu)^{n} T(\varepsilon)(-\partial f(\varepsilon) / \partial \varepsilon)$ is the so-called Lorenz function, $\mu$ denotes the chemical potential and $f(\varepsilon)$ represents the equilibrium Fermi-Dirac function.

The $L_{n}$ quantities can then be used to define the thermoelectric coefficients. The electrical conductance and the electron contribution to the thermal conductance are given by the following expressions, respectively:

$$
\begin{gathered}
G=e^{2} L_{0}, \\
K_{\mathrm{el}}=(1 / T)\left(L_{2}-L_{1}^{2} / L_{0}\right) .
\end{gathered}
$$

In the linear response regime the thermoelectric coefficients are calculated at points where both the bias voltage and temperature gradient between the two electrodes tend to vanish, i.e., $\mu_{\mathrm{L}}=\mu_{\mathrm{R}}=\mu$ and $T_{\mathrm{L}}=T_{\mathrm{R}}=T$.
The thermopower can be expressed as the ratio of the induced voltage difference to the temperature gradient when the current vanishes:

$$
S=-\frac{\Delta V}{\Delta T}=-(1 / e T)\left(L_{1} / L_{0}\right),
$$

The reciprocal interaction between the electrical conductance, thermal conductance and thermopower finally shapes the thermoelectric efficiency of the system, i., e, the figure of merit:

$$
Z T=S^{2} T G /\left(K_{\mathrm{el}}+K_{\mathrm{ph}}\right)
$$

Rigorous theoretical and experimental studies have shown that the thermal conductance in polycyclic organic based molecular junctions with $\mathrm{Au}$ electrodes is notably dominated by electrons $[13,44]$. Therefore, without loss of generality we only considered the electron contribution to the thermal conductance in this study $[7,21]$.

\section{RESULTS}

\subsection{The Unperturbed Molecular Junction}

First, we assumed a situation where the anthracene molecule is attached to the $\mathrm{Au}$ electrodes via three different anchoring groups, i.e., thiol, isocyanide and cyanide units, in the $\mathrm{X}$ position (shown in Figure 1A) and ignored the presence of any side group (i.e., the unperturbed configuration). The choice of anchoring group can determine the nature of charge transport in the system to be either p-type (HOMO-dominated) or n-type (LUMO-dominated) $[21,30,31]$ by the rearrangement of FMOs [28]. In this way, anchoring groups can be discriminated by their electrode-coupling induced charge transfer properties [45].

The calculated transmission spectrum of the molecular junction in the presence of each anchoring group is shown in Figure 2A (on a logarithmic scale). Color-coded H/L labels indicate the HOMO/LUMO transmission peaks corresponding to the anchoring group with the same color: Thiol (red), isocyanide (blue) and cyanide (green). The transmission spectrum of the thiol-terminated anthracene molecular junction shows p-type (HOMO-dominated) transport, whereas the same molecule terminated with isocyanide and cyanide anchoring groups shows n-type (LUMO-dominated) transport. More specifically, when the coupling of the molecule to the $\mathrm{Au}$ electrodes is mediated by the thiol anchoring group (red) the HOMO energy (H-labeled peak in Figure 2A) is located closer to the Fermi energy (p-type transport) than the corresponding LUMO energy (L-labeled peak in Figure $\mathbf{2 A}$ ) due to the electron-donating nature of the thiol unit which increases the energy of the $\pi$-electron system. On the contrary, for the electronaccepting isocyanide (blue) and cyanide (green) anchoring groups, the electron transport occurs through the LUMO (n-type transport) denoted by transmission peaks labeled with the same-color letter L in Figure 2A. These observations are in agreement with the results of previous studies [30, 31]. The behavior of transmission spectrum in the presence of different 

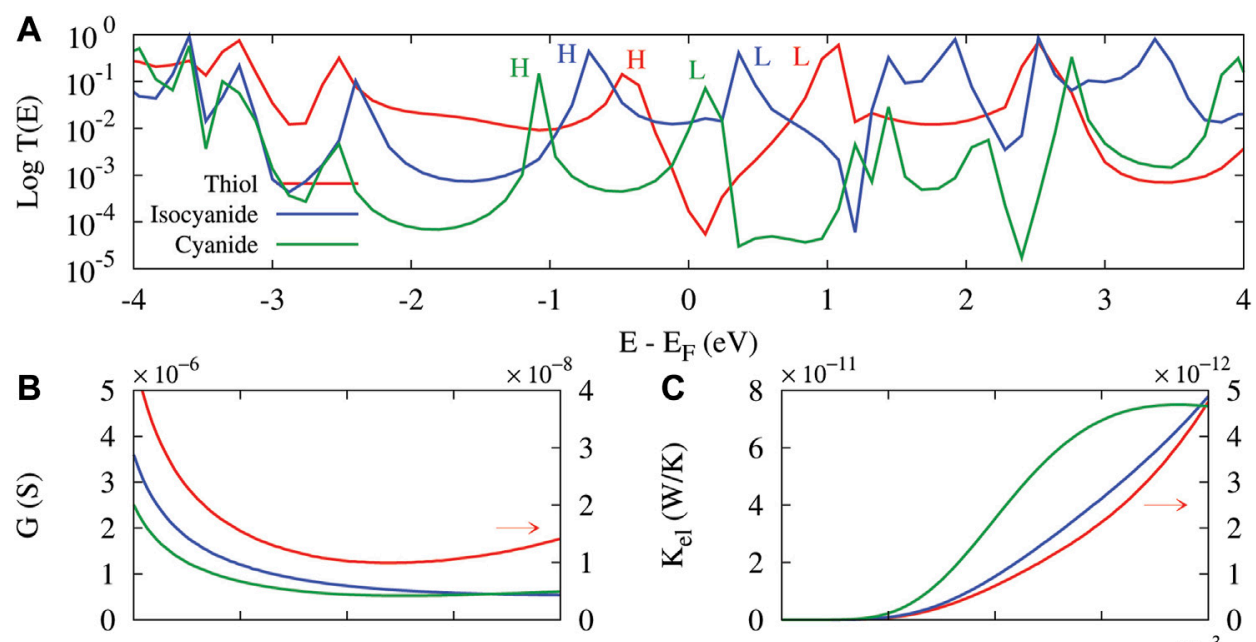

$\mathrm{E}-\mathrm{E}_{\mathrm{F}}(\mathrm{eV})$
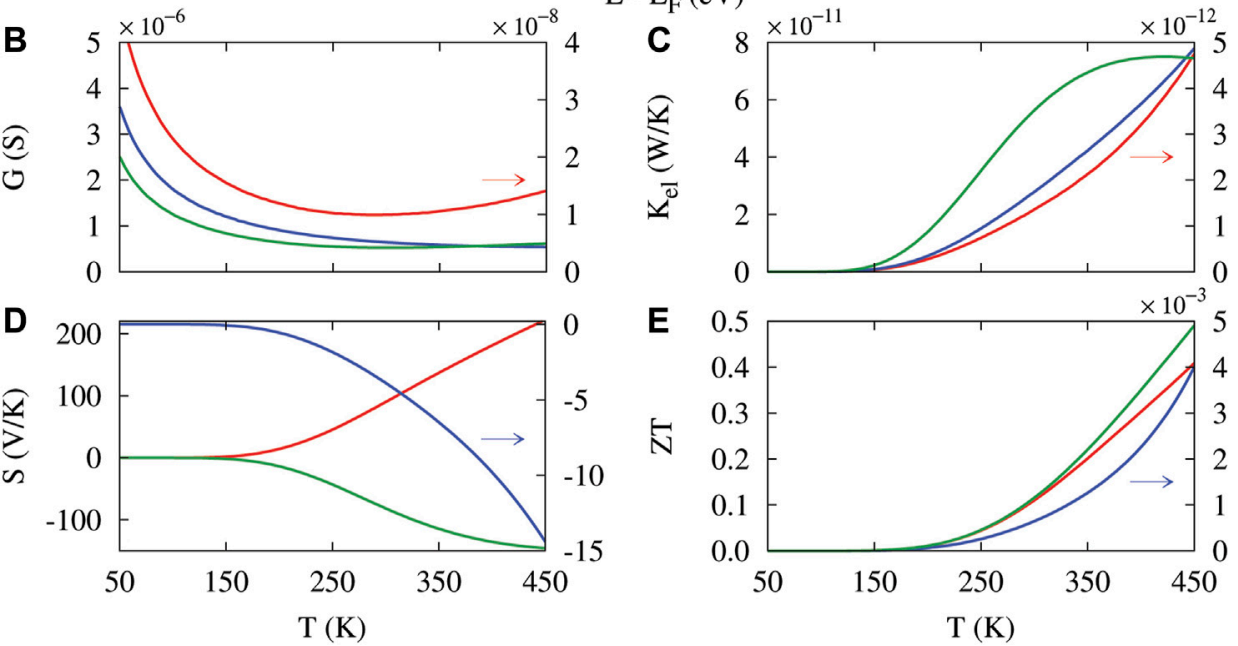

FIGURE 2 | (A) The transmission spectrum versus energy for the unperturbed anthracene molecule coupled to the Au electrodes via thiol (red), isocyanide (blue) and cyanide (green) anchoring groups which was evaluated at $T=300 \mathrm{~K}$. HOMO/LUMO transmission peaks are labeled by letter H/L. Temperature dependency of the electrical conductance (B), electronic thermal conductance (C), thermopower (D) and figure of merit (E) in the unperturbed molecular junction. Colored arrows indicate that the corresponding curve is depicted against y2-axis (right).
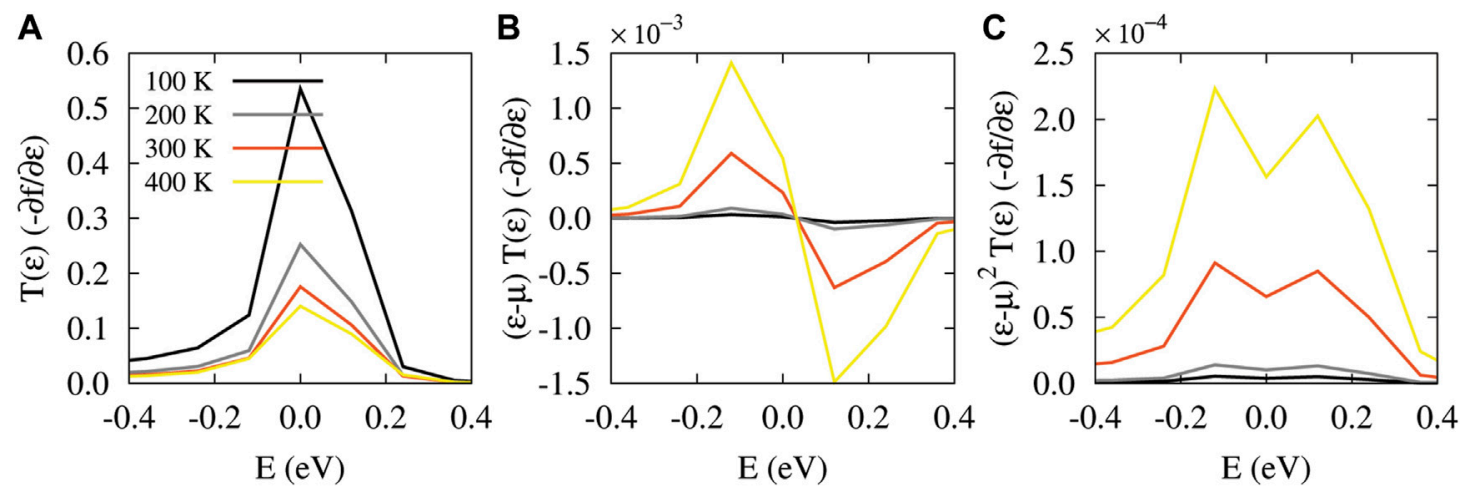

FIGURE 3 The temperature dependency of Lorenz functions is examplary drawn for the unperturbed molecular junction terminated with the thiol anchoring group. (A) $T(\varepsilon)(-\partial f / \partial \varepsilon)$. (B) $(\varepsilon-\mu) T(\varepsilon)(-\partial f / \partial \varepsilon)$. (C) $(\varepsilon-\mu)^{2} T(\varepsilon)(-\partial f / \partial \varepsilon)$.

anchoring groups arises due to the difference between the electrode-coupling induced charge transfer for each anchoring group [45], and the electronegativity of the molecule (mediated by each anchoring group) and that of the Au electrodes [21].

The realignment of the FMOs in the transmission spectrum by anchoring groups determines the dominant carriers participating in transport which is strongly related to the change of chemical potential of the electrodes [46]. This can ultimately shape the temperature dependency of thermoelectric properties of the molecular junction that are depicted in Figures 2B-E. The electrical conductance of the unperturbed molecule for all of the anchoring groups decreases with temperature and relatively saturates at temperatures over $T=300 \mathrm{~K}$ (see Figure 2B). This is due to the temperature-dependent broadening of the Fermi derivative around the Fermi energy. This finally decreases the number of carriers contributing in transport leading to a 
suppressed electrical conductance. The behavior of the electrical conductance can be explained by the temperature-dependent modification of $T(\varepsilon)(-\partial f / \partial \varepsilon)$ associated with the $L_{0}$ term in Eq. 7. As examplary shown in Figure $\mathbf{3 A}$, the $T(\varepsilon)(-\partial f / \partial \varepsilon)$ term for the thiol-terminated molecule is suppressed near the Fermi energy with increasing the temperature leading to a decreased electrical conductance. Similar argument can be brought forward for the isocyanide and cyanide anchoring groups. Notably, the lowest value of the electrical conductance belongs to the thiol-terminate molecule (Figure 2B, red). The behavior of the electrical conductance can be attributed to the location of HOMO and LUMO peaks in the corresponding transmission coefficient of the system in the case of each anchoring group (see Figure 2A).

The electron contribution to the thermal conductance for all of the anchoring groups is increased with temperature (see Figure 2C) since electrons and holes carry more thermal energy when the temperature increases. This observation can be validated by the temperature dependency of $(\varepsilon-\mu)^{2} T(\varepsilon)(-\partial f / \partial \varepsilon)$ depicted in Figure 3C (for the thiol anchoring group) which strongly modifies the behavior of the electron contribution to the thermal conductance via the $L_{2}$ term in Eq. 8. The $(\varepsilon-\mu)^{2} T(\varepsilon)(-\partial f / \partial \varepsilon)$ term is enhanced with increasing the temperature implying an increased electronic thermal conductance where the lowest value belongs to the thiol-terminated molecule. Same argument can be brought forward for the isocyanide and cyanide anchoring groups.

The sign of the thermopower reveals how HOMO and LUMO energy levels are aligned with respect to the Fermi energy level [47]. A positive thermopower is obtained when the HOMO lies closer to the Fermi energy than the LUMO implying a p-type transport, whereas a negative thermopower indicates that the LUMO is located closer to the Fermi energy resulting in a n-type transport. As shown in Figure 2D, when the molecule is terminated with the electron-donating thiol (red) anchoring unit, the thermopower is positive and follows an ascending trend with temperature. However, the electron-accepting isocyanide (blue) or cyanide (green) anchoring group yields a negative thermopower but its magnitude increases with temperature. The temperature dependency of the thermopower can be explained by the contribution of $(\varepsilon-\mu) T(\varepsilon)(-\partial f / \partial \varepsilon)$ shown in Figure 3B which determines the behavior of the thermopower through the $L_{1}$ term in Eq. 9. Figure 3B shows that the $(\varepsilon-\mu) T(\varepsilon)(-\partial f / \partial \varepsilon)$ term is increased around the Fermi energy with temperature for the thiol anchoring group which leads to the enhancement of thermopower. The thermopower of the molecule terminated with isocyanide and cyanide anchoring groups qualitatively follows the same line of arguments. The temperature-dependent increase/decrease of the thermopower occurs in the vicinity of the Fermi energy where the peak of the transmission coefficient is located since thermopower depends on the slope of the transmission function at the Fermi energy.

Finally, the interaction between the electrical conductance, thermal conductance and thermopower shapes the figure of merit of the molecular junction through Eq. 10 as a function of temperature that is shown in Figure 2E. The smallest value of the figure of merit belongs to the isocyanide-terminated system (blue), whereas the cyanide-terminated molecule (green) has the greatest figure of merit. The behavior of the figure of merit is mostly shaped by the thermopower since $Z T \propto S^{2}$, which was most significant for the thiol anchoring group. However, in this case the small electrical conductance of the thiol-terminated system prevented the figure of merit to attain the greatest value.

\subsection{The Molecular Junction Perturbed With the Side Group}

So far, we inspected the thermoelectric properties of the anthracene molecular junction for three different anchoring groups. Next, we will introduce the amine side group to the system, in two different positions labeled as R1 and R2 in Figure 1A (i.e., the perturbed configuration) where the molecule is coupled to the $\mathrm{Au}$ electrodes via previously described anchoring units. The interplay between charge donating/accepting nature of anchoring groups and that of the side group, as well as the position of the side group may strongly modify the thermoelectric properties of the molecular junction [21]. To further explore these modifications, we repeated calculations for the electrical conductance, thermal conductance, thermopower and figure of merit of the system in the presence of the amine side group.

Notably, the perturbation of the molecule with side group can rearrange HOMO or LUMO alignment relative to the Fermi energy, and consequently, modify the thermoelectric properties of the molecular junction depending on the position of the side group. This is illustrated in Figure 4 where the transmission coefficient of the molecular junction perturbed with the amine side group is depicted as a function of energy. In the case of molecule terminated with the thiol anchoring group, the HOMO peak in the transmission spectrum is shifted towards the Fermi energy upon the introduction of the side group (Figure $\mathbf{4 A}$, magenta/cyan curves) in comparison to the unperturbed molecule (red curve). In the isocyanide-terminated molecule the HOMO peaks are slightly shifted closer to the Fermi energy, whereas the LUMO peaks are relatively in the same location when the molecule is perturbed with the side group (Figure 4B, magenta/cyan curves) compared to the unperturbed molecule (Figure 4B, blue curve), leading to a reduced HOMO-LUMO gap. In the cyanide-terminated molecule, the most significant observation is that the introduction of the side group in the $\mathrm{R} 2$ position leads to the emergence of sharp HOMO and LUMO peaks (Figure 4C, cyan curve) in comparison to the unperturbed molecule (Figure 4C, green curve). However, the HOMO and LUMO peaks are slightly suppressed in the R1 position of the side group (Figure 4C, magenta curve). The introduction of the side group crucially modulates the transmission probability near the Fermi energy and shifts the transmission peaks due to constructive/destructive quantum interference effects $[48,49]$ and the interaction between charge donating/accepting nature of the side group and different anchoring groups $[21,24,50]$.

Then, we looked into the behavior of the electrical conductance, thermal conductance, thermopower and figure of merit in the presence of the side group (in two different 

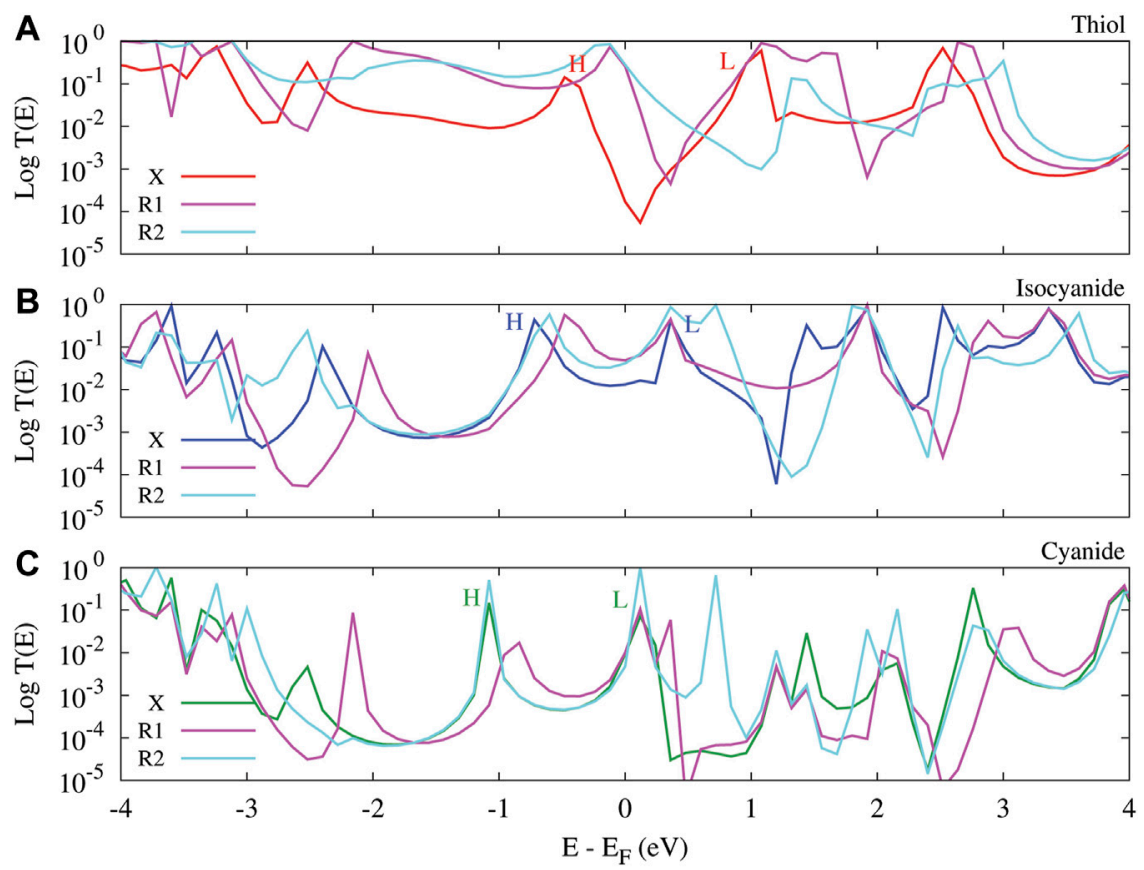

FIGURE 4 | The transmission spectrum versus energy for the anthracene molecule perturbed with the amine side group in R1 and R2 positions which was evaluated at $T=300 \mathrm{~K}$. The molecule was connected to the Au electrodes via thiol (A), isocyanide (B) and cyanide (C) anchoring groups. The transmission of the unperturbed molecular junction (labeled $\mathrm{X}$ ) is depicted as a reference. HOMO/LUMO peaks of the unperturbed system are labeled by letter $\mathrm{H} / \mathrm{L}$.

positions) when the molecule is anchored with thiol, isocyanide and cyanide units. For both the unperturbed molecule (labeled $\mathrm{X}$ for each anchoring group) and the molecule perturbed with the side group in $\mathrm{R} 1$ and $\mathrm{R} 2$ positions, the electrical conductance decreases with temperature (see Figure 5A1-A3), except for the cyanide-terminated molecule with side group in the R2 position (Figure 5A3, cyan curve). As argued earlier, the electrical conductance is decreased since the transmission spectrum is modified accordingly and the Fermi derivative $(-\partial f / \partial \varepsilon)$ associated with $L_{0}$ is broadened around the Fermi energy (not shown). In this case, the overlap between the Fermi derivative and the peak of the transmission is decreased with temperature [21], which restricts the number of carriers contributing in transport implying a decreased electrical conductance. However, when the temperature increases, the Fermi derivative is broadened and more likely embraces additional peaks of the transmission spectrum which enhances the electrical conductance, e.g., in the case of the cyanide-terminated molecule with side group in the R2 position (see Figure 5A3, cyan curve). Notably, the value of the electrical conductance has been relatively increased upon the introduction of the side group (cf. Figures 4A1-A3, red/blue/green and magenta/cyan curves). This side-groupinduced increasing effect can be traced back to the modulation of HOMO and LUMO locations in the corresponding transmission spectrum compared to the unperturbed system.

Similar to the perturbed molecule, the electron contribution to the thermal conductance of the perturbed molecule follows an ascending trend when the temperature is increased (see Figures 5B1-B3). This was expected because charge carriers transfer more thermal energy as the temperature increases. In particular, the introduction of the side group enhanced the temperaturedependent electronic thermal conductance in comparison to the unperturbed molecule for all of the three anchoring groups (cf. Figures 5B1-B3, red/blue/green and magenta/cyan curves). The temperature increasing behavior of the thermal conductance can be explained by the enhanced overlap between the peaks of transmission and Fermi derivative and the contribution of $(\varepsilon-\mu)^{2} T(\varepsilon)(-\partial f / \partial \varepsilon)$ linked with $L_{2}$ term in Eq. 8 that leads to an enhanced thermal conductance with temperature (not shown). Furthermore, for all of the three anchoring groups, changing the position of the side group from R1 to R2 does not induce any major different in overall behavior of the electrical conductance or the thermal conductance, except the cyanide-terminated molecule with the side group in the R2 position (Figures 5A3, B3, cyan curve). The side-group-mediated emergence of new sharp peaks in the transmission spectrum of the cyanide-terminated molecule perturbed with side group in the R2 position (Figure 4C, cyan curve) can account for this different behavior compared to the same system perturbed with side group in the R1 position (Figure 4C, magenta curve).

The role of thermopower in shaping the behavior of the figure of merit is more critical than the electrical conductance or the thermal conductance since the figure of merit quadratically depends on the thermopower. Furthermore, the sign of thermopower indicates the conductance mechanism through the system: Negative (positive) thermopower is a footprint of charge transfer 

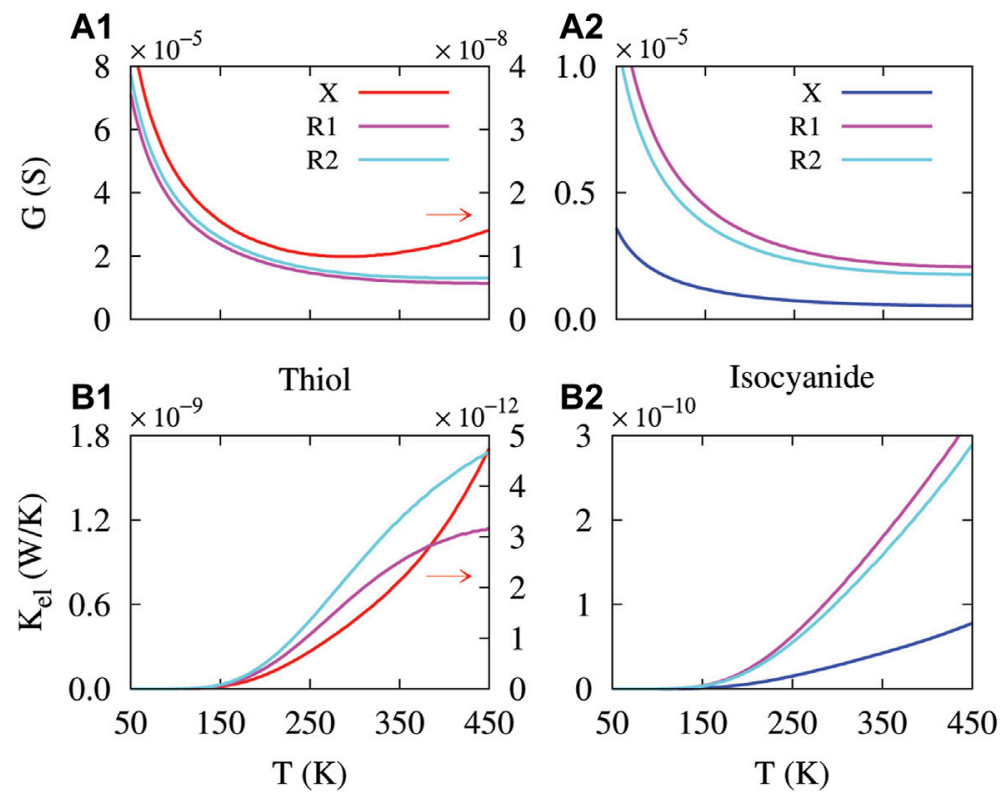
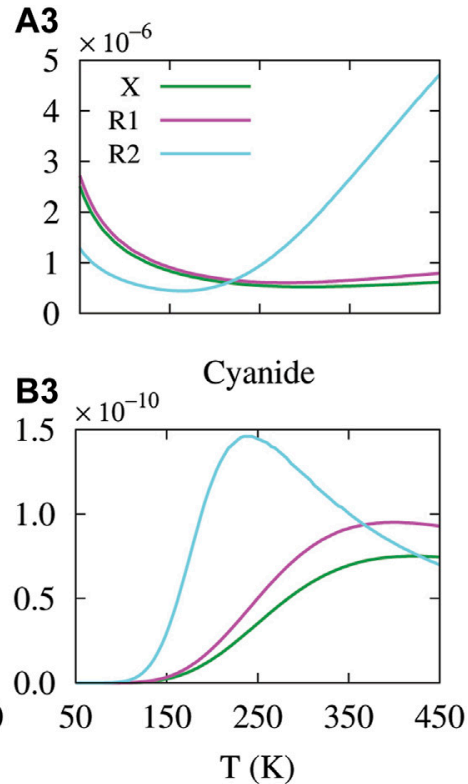

FIGURE 5 | Temperature dependency of the electrical conductance (A1-A3) and electron contribution to the thermal conductance (B1-B3) in the molecular junction perturbed with the amine side group in R1 and R2 positions. The molecule was connected to the Au electrodes via thiol (first column), isocyanide (second column) and cyanide (third column) anchoring groups. The electrical conductance and electronic thermal conductance of the unperturbed molecular junction (labeled X) are depicted as a reference. Colored arrows indicate that the corresponding curve is depicted against y2-axis (right).

dominated by electrons (holes). This interpretation reveals other information about the location of FMOs with respect to the Fermi energy, i.e., negative (positive) thermopower is the indicator of a LUMO-dominated (HOMO-dominated) charge transfer. In this way, charge transfer properties of anchoring groups can strongly determine the sign of the thermopower in the molecular junction [30].

Figure 6A1-A3 shows the thermopower of the perturbed molecular junction. The absolute value of the thermopower is increased with temperature for both the unperturbed and perturbed molecules anchored with the electron-donating thiol (Figure 6A1; positive thermopower) or the electron-accepting isocyanide and cyanide units (Figure 6A2,A3; negative thermopower). The magnitude of the thermopower, however, is determined by the location of HOMO or LUMO peak relative to the Fermi energy in the transmission spectrum. In fact, the thermopower is proportional to the slope of the transmission near the Fermi energy on the logarithmic scale, i.e., $S \propto d \varepsilon \log (T(\varepsilon))$. This explains why the magnitude of thermopower in the thioland cyanide-terminated molecules (Figure 6A1-A3, respectively) is greater than the isocyanide-terminated molecule (Figure 6A2). In the thiol-terminated molecule, the introduction of the side group reduced the magnitude of thermopower (cf. Figure 6A1, red and magenta/cyan curves). However, in the isocyanide- and cyanide-terminated molecules the presence of the side group in the R2 position notably increased the temperature dependency of the thermopower (cf. Figure 6A2,A3, red/magenta and cyan curves). The behavior of the thermopower in either of these cases can be explained by the temperature dependency of the $(\varepsilon-\mu) T(\varepsilon)$ $(-\partial f / \partial \varepsilon)$ term (not shown) that is related to $L_{1}$ in Eq. 9 which ultimately shapes the trend of the thermopower. However, while the sign of the thermopower is determined by the charge transport nature of the anchoring group and side group, its magnitude is determined by the location of HOMO and LUMO peaks relative to the Fermi energy in the transmission spectrum.

Ultimately, the electrical conductance, thermal conductance and thermopower compete to determine the thermoelectric figure of merit of the molecular junction given by Eq. 10. The temperature dependency of the figure of merit for all considered configurations is depicted in Figures 6B1-B3. In general, the lowest value of the figure of merit belongs to the isocyanide-terminated molecule (Figure 6B2), whereas the greatest value of the figure of merit belongs to the cyanideterminated molecule (Figure 6B3). Notably, at high temperatures the figure of merit of the cyanide-terminated molecule perturbed with the side group in the $\mathrm{R} 2$ position can attain high values, i.e., $Z T \sim 1$. In particular, perturbation of the molecule with the side group (regardless of the its position) decreases the figure of merit of the thiol-terminated system (cf. Figure 6B1, red and magenta/cyan curves). There was no major difference between the R1 and R2 position of the side group in this case as they both yielded relatively similar values for the figure of merit (see Figure 6B1, magenta/cyan curves). In the isocyanide-terminated molecule, however, the introduction of the side group in the $\mathrm{R} 1$ position slightly reduced the figure of merit (Figure 6B2, magenta curve) in comparison to the unperturbed molecule (Figure 6B2, blue curve). The side group in the R2 position increased the figure of merit in this case (Figure 6B2, cyan curve). In the cyanide-terminated molecule, the 

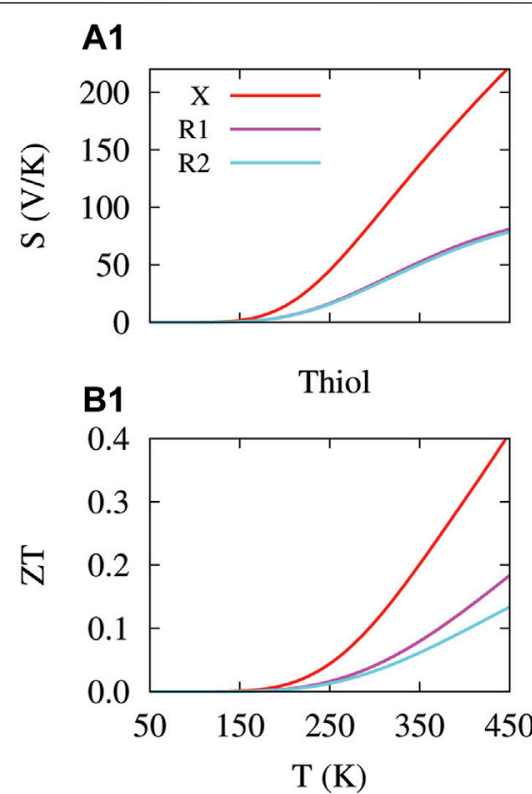
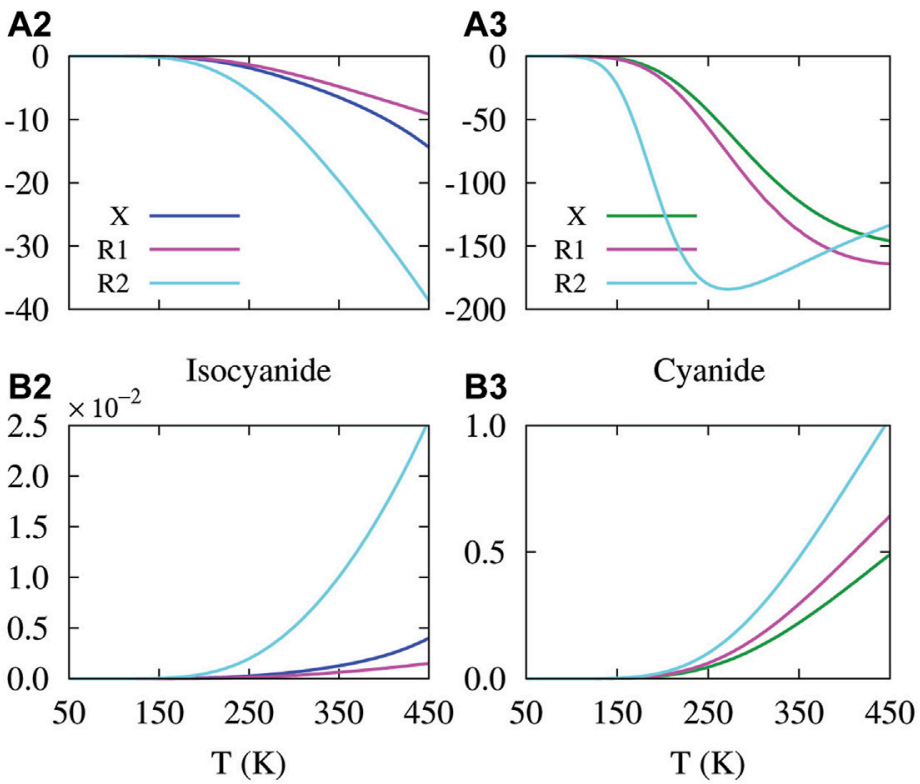

FIGURE 6 | Temperature dependency of the thermopower (A1-A3) and figure of merit (B1-B3) in the molecular junction perturbed with the amine side group in R1 and R2 positions. The molecule was connected to the Au electrodes via thiol (first column), isocyanide (second column) and cyanide (third column) anchoring groups. The thermopower and figure of merit of the unperturbed molecular junction (labeled X) are depicted as a reference.

introduction of the side group in both positions shifted the figure of merit towards greater values (Figure 6B3, magenta/cyan curves) compared to the unperturbed molecule (Figure 6B3, green curve).

\subsection{Comparison Between the Unperturbed and Perturbed Molecules}

To compare the influence of the charge transfer nature of anchoring groups and the side group position on the electrical conductance, thermal conductance, thermopower and figure of merit in the anthracene molecular junction, the results were summarized in Figure 7 where the calculations were carried out at $T=300 \mathrm{~K}$. Figure 7A shows that the electrical conductance trend is as follows: In the unperturbed molecule: thiol $<$ cyanide $<$ isocyanide, in the molecule perturbed with the side group in the $\mathrm{R} 1$ position: cyanide $<$ thiol < isocyanide, and in the $\mathrm{R} 2$ position: thiol $<$ cyanide $<$ isocyanide. The greatest value of the electrical conductance at $T=300 \mathrm{~K}$ belonged to the isocyanide-terminated molecule perturbed with the side group in the $\mathrm{R} 1$ position, i.e., $G=2.42 \times 10^{-6} \mathrm{~S}$. The trend of thermal conductance, however, is as follows: In the unperturbed molecule: thiol < isocyanide < cyanide, in the molecule perturbed with the side group in the R1 position: cyanide $<$ isocyanide $<$ thiol, and in the R2 position: isocyanide < cyanide < thiol. The greatest value of the thermal conductance at $T=300 \mathrm{~K}$ belonged to the thiolterminated molecule perturbed with the side group in the R2 position, i.e., $K_{\mathrm{el}}=8.54 \times 10^{-11} \mathrm{~W} / K$.

The thermopower showed the following trend illustrated in Figure 7C: In the unperturbed molecule: isocyanide $<$ cyanide $<$ thiol, in the molecule perturbed with the side group in the R1 position: isocyanide $<$ thiol $<$ cyanide, and in the R2 position: isocyanide $<$ thiol $<$ cyanide. The cyanide-terminated molecule perturbed with the amine side group in the R2 positions attained the greatest thermopower at $T=300 \mathrm{~K}$, i.e., $S=180.38 \mathrm{~V} / \mathrm{K}$. Figure 7D shows the figure of merit of the system evaluated at $T=300 \mathrm{~K}$ which qualitatively follows the trend of thermopower: In the unperturbed molecule: isocyanide $<$ thiol < cyanide, in the molecule perturbed with the side group in the R1 position: isocyanide < thiol < cyanide, and in the R2 position: isocyanide $<$ thiol < cyanide. Notably, the cyanide-terminated molecule perturbed with the amine side group in the R2 position reached the greatest value of the figure of merit at $T=300 \mathrm{~K}$, i.e., $Z T=0.25$.

\subsection{Violation of the Wiedemann-Franz Law}

It was predicted that the Wiedemann-Franz law holds for certain bulk metals, however, its validity is questioned when the dimension is reduced to the nanoscale. Recently, the thermal and electrical transports were precisely measured in atomic-sized contacts by using advanced experimental methods [51, 52]. The validity of the Wiedemann-Franz law was substantiated at this scale. But, extremely thorough and careful theoretical studies raised serious doubts about the generality of such observations and argued that significant deviations may arise depending on the structure of the system, temperature or gate voltage [53].

To address such concerns in the context of our study, we quantitatively checked the validity of the Wiedemann-Franz law for the Au-anthracene-Au molecular junction. The Lorenz ratio is defined as follows [44]:

$$
\frac{\mathcal{L}}{\mathcal{L}_{0}}=\frac{K_{\mathrm{el}}}{\mathcal{L}_{0} T G}
$$



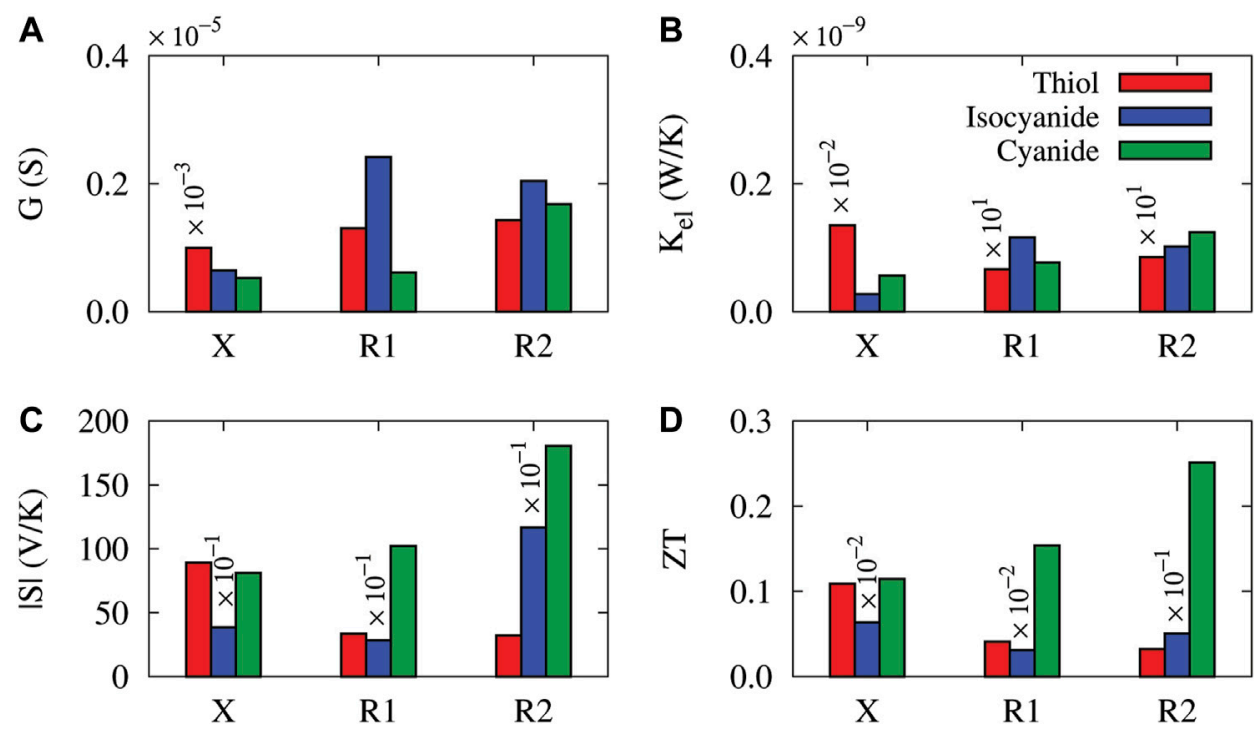

FIGURE 7 | The electrical conductance (A), electronic thermal conductance (B), absolute value of the thermopower (C) and figure of merit (D) of the unperturbed molecular junction (labeled X), and the molecule perturbed with the amine side group in R1 and R2 positions are compared at $T=300 \mathrm{~K}$. The coupling of the molecule to the Au electrodes was mediated by thiol (red), isocyanide (blue) and cyanide (green) anchoring groups.

where $\mathcal{L}$ is the Lorenz number that converges to the Sommerfeld value, i.e., $\mathcal{L}_{0}=\pi^{2} k_{\mathrm{B}}^{2} /\left(3 e^{2}\right)=2.44 \times 10^{-8} \mathrm{~W} / S K^{2}$, when the Wiedemann-Franz law holds (i.e., $\mathcal{L} / \mathcal{L}_{0}=1$ ).

We then calculated the temperature dependency of Lorenz ratios (i.e., $\mathcal{L} / \mathcal{L}_{0}$ ) defined in Eq. 11 for the unperturbed molecule (i.e., without side group) and the perturbed molecule (i.e., with the amine side group in the R1 and R2 positions), both anchored with the thiol, isocyanide and cyanide units. This is illustrated in Figure 8 indicating that the Wiedemann-Franz law is violated in the Au-anthracene-Au molecular junction, irrespective of the presence or position (i.e., R1 vs R2 in Figures 8A-C) of the side group and regardless of the type of the anchoring group (i.e., thiol (red), isocyanide (blue) and cyanide (green) in the $\mathrm{X}$ position shown in Figures 8A-C).

It has been predicted that deviations from the WiedemannFranz law (i.e., $\mathcal{L} / \mathcal{L}_{0} \neq 1$ ) in metallic atomic-size contacts may arise due to significant phonon contributions [44, 54]. However, in polycyclic organic molecular junctions with Au electrodes the thermal conductance is significantly dominated by electrons [13, 44]. Alternatively, it was suggested that quantum interference effects and energy dependency of the transmission spectrum in the vicinity of the Fermi energy are responsible for the notable violation of the Wiedemann-Franz law in organic molecular structures with $\mathrm{Au}$ electrodes [53], as observed in our study. In fact, as shown in Figures 4A-C, non-smooth dependence of the transmission coefficient on energy near the Fermi level leads to the enhancement of the electronic thermal conductance with temperature due to the temperature-dependent increase of $(\varepsilon-\mu)^{2} T(\varepsilon)(-\partial f / \partial \varepsilon)$ vs the descending behavior of the electrical conductance with temperature due to the temperature-dependent reduction of $T(\varepsilon)(-\partial f / \partial \varepsilon)$. The nonidentical modulation of the electronic thermal conductance and the electrical conductance finally promoted the calculated
Lorenz ratio leading to temperature-dependent deviations from the Wiedemann-Franz law (see Figure 8).

\section{DISCUSSION}

In the present study, we theoretically explored the thermoelectric properties of an anthracene-based molecular device with $\mathrm{Au}$ electrodes by using wide-band (WB) approximation combined with the NEGF formalism in the linear response regime. Specifically, our intention was to address the question that how the electrical conductance, thermal conductance, thermopower and figure of merit of the Au-anthracene-Au molecular structure can be shaped when the system is terminated with different anchoring groups or it is perturbed with a side group in comparison to the unperturbed molecule (i.e., without side groups). We considered one side group but in two different positions indicated by R1 and R2 and comparatively examined the behavior of thermoelectric coefficients in the presence of three anchoring groups. The results indicated that modification of the Au-anthracene-Au molecular junction with different anchoring groups or with a side group in different positions can significantly change the thermoelectric properties of the system.

We assumed an electron-donating side group (amine) in two different positions for the functionalization of the molecule where the molecule-electrode coupling was mediated by both electrondonating (thiol) and electron-accepting (isocyanide, cyanide) anchoring groups. We found that the charge transfer nature of the system (dictated by each anchoring group) remains intact to the perturbation of the molecule with the side group. In this way, termination of the molecule with the electron-donating thiol unit led to a HOMO-dominated transmission, whereas when the 

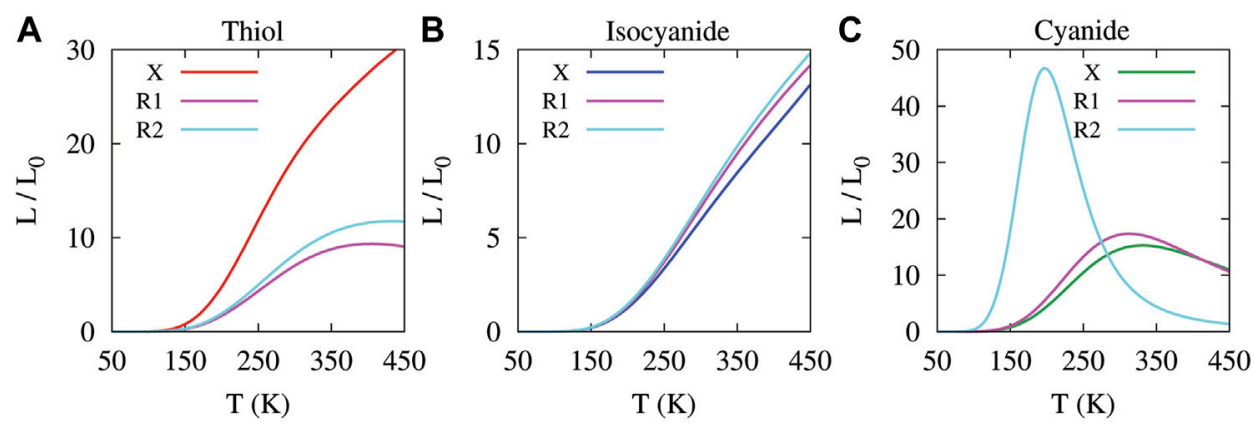

FIGURE 8 | Lorenz ratios defined by Eq. 11 as a function of temperature for the unperturbed (labeled X) molecule and the molecule perturbed with the amine side group in R1 and R2 positions. The coupling of the molecule to the Au electrodes was mediated by thiol (A), isocyanide (B) and cyanide (C) anchoring groups.

molecule was anchored with the electron-accepting isocyanide and cyanide units the transmission was LUMO-dominated, irrespective of the side group position. The LUMO peaks were closer to the Fermi energy in the cyanide-anchored molecule than the isocyanide-terminated molecule, implying stronger electronaccepting character of the cyanide group. However, employing electron-accepting side groups such as nitro unit $\left(-\mathrm{NO}_{2}\right)$ may disturb this deduction, as addressed by previous studies [21]. In addition, the main impact of the side group was shifting the location of HOMO/LUMO peaks in the transmission spectrum in comparison to the unperturbed molecule (see Figure 4).

Previous studies showed that charge transfer nature or position of anchoring groups can crucially shape conductance, thermopower and figure of merit of molecular junctions by the realignment of FMOs with respect to the Fermi energy level in the transmission spectrum [30, 31]. By the same token, chemical character or conformational changes of side groups can also result in substantial variations of the transmission spectrum [19-21]. In this way, charge transport polarization through the molecule can be controlled where its consequences are finally reflected in the conductance, thermopower and figure of merit of the system [5, 18-20, 55]. In addition, here the moleculeelectrode contact was mediated by anchoring groups only in one geometrical position. However, similar studies demonstrated that changing the position of the anchoring group can also adjust transport properties of the system by changing the HOMO-LUMO symmetry in the spatial distribution of the molecular orbitals [24, 56]. This finally can manipulate electron transport through the molecular junction due to symmetry-allowed or symmetry-forbidden transport states. Moreover, here we merely assumed two geometrical position for the side group. But, assigning more degrees of freedom to the spatial localization of the side group [55] or rotational variations of the side group relative to the molecular backbone $[5,20]$ can be further a determinant of the thermopower and figure of merit in molecular junctions.

Here, we employed a formalism where only linear effects were taken into account. However, nonlinear effects could, at least in principle, affect the thermoelectric properties of the considered molecular junction. This may happen by inducing a notable temperature or chemical potential difference between the electrodes. In addition, the presence of local electric fields around the different functional groups and/or in proximity of the $\mathrm{Au}$ atoms may play a role in shaping the underlying chemistry, as proven in some recent studies [57].

In this study, we assumed a combination of three anchoring groups and one side group (in two positions) comprising six distinct configurations. Our results showed that electrondonating/accepting nature of anchoring groups modifies the transmission probability near the Fermi level and plays an key role in shaping conductance, thermopower and figure of merit of the molecular junction. These properties can be further adjusted by perturbing the molecule with side groups in different positions. In particular, a comparison at the room temperature showed that the isocyanide-anchored molecule perturbed with the side group in the $\mathrm{R} 1$ position reaches the greatest value of electrical conductance. This may be due to the weak binding strength of the isocyanide to the $\mathrm{Au}$ surfaces which enables isocyanide unit to be adsorbed onto the Au surfaces [58]. This finally enhances the transmission probability near the Fermi energy leading to a greater conductance [59]. However, the greatest value of the thermal conductance belonged to the thiol-terminated molecule perturbed with the side group in the $\mathrm{R} 2$ position, probably due to the strong binding of the thiol unit to the Au surfaces [60]. Notably, the molecule anchored with cyanide (stronger electron-accepting anchoring group) and perturbed with the side group in the $\mathrm{R} 2$ position attained the greatest value of both the thermopower and figure of merit. As it can be seen from the trend of the cyanide-terminated molecule in Figures 7C,D (green boxes), introducing the side group and then shifting its position from R1 to R2 favorably enhances both the thermopower and figure of merit. At the room temperature, in the best scenario, the figure of merit reaches $Z T=0.25$.

The results presented in this study are in agreement with experimental findings obtained in organic molecular junctions sandwiched between two metallic electrodes. For instance, experimental measurements on metal-molecule-metal junctions revealed that the electrical conductance is crucially modified by anchoring groups $[14,22,61]$ or side groups [36, $38,39]$. However, inability to simultaneously measure the electrical, thermal and thermoelectric properties of singlemolecule junctions may be one of the technical challenges of 
the traditional experimental methods. This limitation was overcame in several experiments reporting, e.g., simultaneous measurement of the electrical conductance and thermopower in single-molecule devices based on break junction techniques $[10,62,63]$. It was not until recently that Mosso et al. simultaneously measured the electrical conductance and thermal conductance of organic molecular junctions with $\mathrm{Au}$ electrodes via a modified break junction technique [13]. Such experimental techniques render the possibility of careful inspection of the Wiedemann-Franz law in organic singlemolecule junctions that has been considered in this study. Thus, our findings may enable an understanding of the role of functionalization in shaping the electrical and thermoelectric properties of organic molecular junctions which may encourage experimentalists to further examine the validity of the Wiedemann-Franz law and structure-function properties of single-molecule junctions.

Ultimately, a potential application of organic molecular junctions is the development of synaptic devices for brain-like bioelectronic computing systems [64, 65]. In particular, realization of long-term memory within single-molecule junction synapses based on conductance changes [66] opened a new window towards implementation of brain-like synaptic plasticity mechanisms into molecular junctions with metal electrodes. In this context, the long-term memory in the device conductance can be manipulated by successive application of appropriate electrical pulses; a counterpart of the spike-timing-dependent plasticity in the brain that modifies the synaptic strengths between neurons [67, 68]. At

\section{REFERENCES}

1. DiSalvo FJ. Thermoelectric Cooling and Power Generation. Science (1999) 285: 703-6. doi:10.1126/science.285.5428.703

2. Bell LE. Cooling, Heating, Generating Power, and Recovering Waste Heat with Thermoelectric Systems. Science (2008) 321:1457-61. doi:10.1126/ science. 1158899

3. Long MA, and Fee MS. Using Temperature to Analyse Temporal Dynamics in the Songbird Motor Pathway. Nature (2008) 456:189-94. doi:10.1038/ nature 07448

4. Aronov D, and Fee MS. Analyzing the Dynamics of Brain Circuits with Temperature: Design and Implementation of a Miniature Thermoelectric Device. J Neurosci Methods (2011) 197:32-47. doi:10.1016/j.jneumeth.2011.01.024

5. Finch C, Garcia-Suarez V, and Lambert C. Giant Thermopower and Figure of merit in Single-Molecule Devices. Phys Rev B (2009) 79:033405. doi:10.1103/ physrevb.79.033405

6. Nozaki D, Sevinçli H, Li W, Gutiérrez R, and Cuniberti G. Engineering the Figure of merit and Thermopower in Single-Molecule Devices Connected to Semiconducting Electrodes. Phys Rev B (2010) 81:235406. doi:10.1103/ physrevb.81.235406

7. Ramezani Akbarabadi S, Rahimpour Soleimani H, Golsanamlou Z, and Bagheri Tagani M. Enhanced Thermoelectric Properties in Anthracene Molecular Device with Graphene Electrodes: the Role of Phononic thermal Conductance. Sci Rep (2020) 10:10922-13. doi:10.1038/s41598020-67964-w

8. Torres A, Pontes RB, da Silva AJR, and Fazzio A. Tuning the Thermoelectric Properties of a Single-Molecule junction by Mechanical Stretching. Phys Chem Chem Phys (2015) 17:5386-92. doi:10.1039/c4cp04635h higher levels, these memory devices can be integrated in crossbar arrays [69] representing the strength of plastic synaptic connections in brain neural networks [70, 71]. The results presented here may contribute to further understanding of how the conductance of such molecular structures could be modified/controlled by the modification of anchoring groups or side groups. In fact, side groups in the perturbed molecule can be regarded as a chemical gate that determine the transmission probability or shift the peaks of the transmission spectrum relative to the Fermi energy in comparison to the unperturbed molecule. In this way, the conductance of the system can be tuned to attain stable and favorable regimes.

\section{DATA AVAILABILITY STATEMENT}

The original contributions presented in the study are included in the article/Supplementary Material, further inquiries can be directed to the corresponding author.

\section{AUTHOR CONTRIBUTIONS}

SR and MM contributed to conception and design of the study. SR performed the numerical simulations and theoretical approximations. SR and MM analyzed the results. SR and MM wrote the first draft of the manuscript. All authors contributed to manuscript revision, read, and approved the submitted version.

9. Tsutsui M, Morikawa T, He Y, Arima A, and Taniguchi M. High Thermopower of Mechanically Stretched Single-Molecule Junctions. Sci Rep (2015) 5:1-9. doi:10.1038/srep11519

10. Widawsky JR, Darancet P, Neaton JB, and Venkataraman L. Simultaneous Determination of Conductance and Thermopower of Single Molecule Junctions. Nano Lett (2012) 12:354-8. doi:10.1021/nl203634m

11. Baheti K, Malen JA, Doak P, Reddy P, Jang S-Y, Tilley TD, et al.Probing the Chemistry of Molecular Heterojunctions Using Thermoelectricity. Nano Lett (2008) 8:715-9. doi:10.1021/nl0727381

12. Reddy P, Jang S-Y, Segalman RA, and Majumdar A. Thermoelectricity in Molecular Junctions. Science (2007) 315:1568-71. doi:10.1126/science.1137149

13. Mosso N, Sadeghi H, Gemma A, Sangtarash S, Drechsler U, Lambert C, et al.Thermal Transport through Single-Molecule Junctions. Nano Lett (2019) 19:7614-22. doi:10.1021/acs.nanolett.9b02089

14. Tan A, Balachandran J, Sadat S, Gavini V, Dunietz BD, Jang S-Y, et al.Effect of Length and Contact Chemistry on the Electronic Structure and Thermoelectric Properties of Molecular Junctions. J Am Chem Soc (2011) 133:8838-41. doi:10.1021/ja202178k

15. Ramezani Akbarabadi S, Golsanamlou Z, and Rahimpour Soleimani $\mathrm{H}$. Study of Length-dependent Tunneling Magnetoresistance in Two Phenyl Based Molecules. Curr Phys Chem (2014) 4:285-9. doi:10.2174/ 1877946805666150311234229

16. Pauly F, Viljas JK, and Cuevas JC. Length-dependent Conductance and Thermopower in Single-Molecule Junctions of Dithiolated Oligophenylene Derivatives: A Density Functional Study. Phys Rev B (2008) 78:035315. doi:10.1103/physrevb.78.035315

17. Hüser F, and Solomon GC. From Chemistry to Functionality: Trends for the Length Dependence of the Thermopower in Molecular Junctions. J Phys Chem C (2015) 119:14056-62. doi:10.1021/acs.jpcc.5b04106 
18. Taylor J, Brandbyge $M$, and Stokbro K. Conductance Switching in a Molecular Device: The Role of Side Groups and Intermolecular Interactions. Phys Rev B (2003) 68:121101. doi:10.1103/ physrevb.68.121101

19. Mai C-K, Schlitz RA, Su GM, Spitzer D, Wang X, Fronk SL, et al.Side-chain Effects on the Conductivity, Morphology, and Thermoelectric Properties of Self-Doped Narrow-Band-gap Conjugated Polyelectrolytes. J Am Chem Soc (2014) 136:13478-81. doi:10.1021/ja504284r

20. Papadopoulos TA, Grace IM, and Lambert CJ. Control of Electron Transport through Fano Resonances in Molecular Wires. Phys Rev B (2006) 74:193306. doi:10.1103/physrevb.74.193306

21. Ramezani Akbarabadi S, Rahimpour Soleimani H, and Bagheri Tagani M. Side-group-mediated Thermoelectric Properties of Anthracene SingleMolecule junction with Anchoring Groups. Sci Rep (2021) 11:8958-18. doi:10.1038/s41598-021-88297-2

22. Zotti LA, Kirchner T, Cuevas J-C, Pauly F, Huhn T, Scheer E, et al.Revealing the Role of Anchoring Groups in the Electrical Conduction through Single-Molecule Junctions. Small (2010) 6: 1529-35. doi:10.1002/smll.200902227

23. Chen F, Li X, Hihath J, Huang Z, and Tao N. Effect of Anchoring Groups on Single-Molecule Conductance: Comparative Study of Thiol-, Amine-, and Carboxylic-Acid-Terminated Molecules. J Am Chem Soc (2006) 128:15874-81. doi:10.1021/ja065864k

24. Koga J, Tsuji Y, and Yoshizawa K. Orbital Control of Single-Molecule Conductance Perturbed by $\pi$-Accepting Anchor Groups: Cyanide and Isocyanide. J Phys Chem C (2012) 116:20607-16. doi:10.1021/jp3068156

25. Akbarabadi SR, Soleimani HR, Tagani MB, and Golsanamlou Z. Impact of Coupling Geometry on Thermoelectric Properties of Oligophenyl-Base Transistor. Chin Phys B (2017) 26:027303. doi:10.1088/1674-1056/26/2/ 027303

26. Cao XH, Zhou WX, Chen CY, Tang LM, Long M, and Chen KQ. Excellent Thermoelectric Properties Induced by Different Contact Geometries in Phenalenyl-Based Single-Molecule Devices. Sci Rep (2017) 7:10842-8. doi:10.1038/s41598-017-11224-x

27. Zhang G, and Li B. Impacts of Doping on thermal and Thermoelectric Properties of Nanomaterials. Nanoscale (2010) 2:1058-68. doi:10.1039/ c0nr00095g

28. Stadler R, and Jacobsen KW. Fermi Level Alignment in Molecular Nanojunctions and its Relation to Charge Transfer. Phys Rev B (2006) 74: 161405. doi:10.1103/physrevb.74.161405

29. Karthäuser S. Control of Molecule-Based Transport for Future Molecular Devices. J Phys Condens Matter (2010) 23:1-16. doi:10.1088/0953-8984/23/1/ 013001

30. Balachandran J, Reddy P, Dunietz BD, and Gavini V. End-group-induced Charge Transfer in Molecular Junctions: Effect on Electronic-Structure and Thermopower. J Phys Chem Lett (2012) 3:1962-7. doi:10.1021/ jz300668c

31. Balachandran J, Reddy P, Dunietz BD, and Gavini V. End-group Influence on Frontier Molecular Orbital Reorganization and Thermoelectric Properties of Molecular Junctions. J Phys Chem Lett (2013) 4:3825-33. doi:10.1021/ jz401678m

32. Bürkle M, Zotti LA, Viljas JK, Vonlanthen D, Mishchenko A, Wandlowski $\mathrm{T}$, et al.Ab Initiostudy of the Thermopower of Biphenyl-Based SingleMolecule Junctions. Phys Rev B (2012) 86:115304. doi:10.1103/ physrevb.86.115304

33. Xue Y, and Ratner MA. End Group Effect on Electrical Transport through Individual Molecules: A Microscopic Study. Phys Rev B (2004) 69:085403. doi:10.1103/physrevb.69.085403

34. Noori MD, Sangtarash S, and Sadeghi H. The Effect of Anchor Group on the Phonon thermal Conductance of Single Molecule Junctions. Appl Sci (2021) 11:1066. doi:10.3390/app11031066

35. Dekkiche H, Gemma A, Tabatabaei F, Batsanov AS, Niehaus T, Gotsmann B, et al.Electronic Conductance and Thermopower of Single-Molecule Junctions of Oligo(phenyleneethynylene) Derivatives. Nanoscale (2020) 12:18908-17. doi:10.1039/d0nr04413

36. Huang M-L, Zhang F, Wang C, Zheng J-F, Mao H-L, Xie H-J, et al.Side-group Effect on Electron Transport of Single Molecular Junctions. Micromachines (2018) 9:234. doi:10.3390/mi9050234
37. Klöckner JC, Cuevas JC, and Pauly F. Tuning the thermal Conductance of Molecular Junctions with Interference Effects. Phys Rev B (2017) 96:245419. doi:10.1103/physrevb.96.245419

38. Ismael AK, Wang K, Vezzoli A, Al-Khaykanee MK, Gallagher HE, Grace IM, et al.Side-Group-Mediated Mechanical Conductance Switching in Molecular Junctions. Angew Chem Int Ed (2017) 56:15378-82. doi:10.1002/ anie.201709419

39. Huang JR, Huang H, Tao CP, Zheng JF, Yuan Y, Hong ZW, et al.Controlling Contact Configuration of Carboxylic Acid-Based Molecular Junctions through Side Group. Nanoscale Res Lett (2019) 14:253-7. doi:10.1186/s11671-0193087-7

40. Liu C, Kaneko S, Komoto Y, Fujii S, and Kiguchi M. Highly Conductive Single Naphthalene and Anthracene Molecular junction with Well-Defined Conductance. Appl Phys Lett (2015) 106:103103. doi:10.1063/1.4914501

41. Zahid F, Paulsson M, and Datta S. Electrical Conduction through Molecules. Advanced Semiconductor And Organic Nano-Techniques. Cambridge, Massachusetts, United States: Elsevier (2003):1-41. doi:10.1016/b978012507060-7/50022-2

42. Meir Y, and Wingreen NS. Landauer Formula for the Current through an Interacting Electron Region. Phys Rev Lett (1992) 68:2512-5. doi:10.1103/ physrevlett.68.2512

43. Datta S. Quantum Transport: Atom to Transistor. New York, United States: Cambridge University Press (2005)

44. Klöckner JC, Matt M, Nielaba P, Pauly F, and Cuevas JC. Thermal Conductance of Metallic Atomic-Size Contacts: Phonon Transport and Wiedemann-Franz Law. Phys Rev B (2017) 96:205405. doi:10.1103/ physrevb.96.205405

45. Hong S, Reifenberger R, Tian W, Datta S, Henderson JI, and Kubiak CP. Molecular Conductance Spectroscopy of Conjugated, Phenyl-Based Molecules on $\mathrm{Au}(111)$ : the Effect of End Groups on Molecular Conduction. Superlattices and Microstructures (2000) 28:289-303. doi:10.1006/spmi.2000.0916

46. Lin GM, Lin CH, Peng HH, Hsiao H, Wang TH, Ho CH, et al.Effect of the Chemical Potentials of Electrodes on Charge Transport across Molecular Junctions. The J Phys Chem C (2019) 123:22009-17. doi:10.1021/ acs.jpcc.9b05927

47. Liu Y-S, and Chen Y-C. Seebeck Coefficient of Thermoelectric Molecular Junctions: First-Principles Calculations. Phys Rev B (2009) 79:193101. doi:10.1103/physrevb.79.193101

48. Stadler R. Quantum Interference Effects in Electron Transport through Nitrobenzene with Pyridil Anchor Groups. Phys Rev B (2009) 80:125401. doi:10.1103/physrevb.80.125401

49. Markussen T, Stadler R, and Thygesen KS. The Relation between Structure and Quantum Interference in Single Molecule Junctions. Nano Lett (2010) 10: 4260-5. doi:10.1021/nl101688a

50. Huang Y, Zhang M, Ye L, Guo X, Han CC, Li Y, et al.Molecular Energy Level Modulation by Changing the Position of Electron-Donating Side Groups. J Mater Chem (2012) 22:5700-5. doi:10.1039/c2jm16474d

51. Mosso N, Drechsler U, Menges F, Nirmalraj P, Karg S, Riel H, et al.Heat Transport through Atomic Contacts. Nat Nanotech (2017) 12:430-3. doi:10.1038/nnano.2016.302

52. Cui L, Jeong W, Hur S, Matt M, Klöckner JC, Pauly F, et al.Quantized thermal Transport in Single-Atom Junctions. Science (2017) 355:1192-5. doi:10.1126/ science.aam6622

53. Bürkle M, and Asai Y. How to Probe the Limits of the Wiedemann-Franz Law at Nanoscale. Nano Lett (2018) 18:7358-61. doi:10.1021/acs.nanolett.8b03651

54. Balachandran V, Bosisio R, and Benenti G. Validity of the Wiedemann-Franz Law in Small Molecular Wires. Phys Rev B (2012) 86. doi:10.1103/ physrevb.86.035433

55. Bilan S, Zotti LA, Pauly F, and Cuevas JC. Theoretical Study of the Charge Transport through C60-Based Single-Molecule Junctions. Phys Rev B (2012) 85:205403. doi:10.1103/physrevb.85.205403

56. Tsuji Y, Staykov A, and Yoshizawa K. Orbital Views of Molecular Conductance Perturbed by Anchor Units. J Am Chem Soc (2011) 133:5955-65. doi:10.1021/ ja111021e

57. Mollica Nardo V, Cassone G, Ponterio RC, Saija F, Sponer J, and Tommasini M. Electric-Field-Induced Effects on the Dipole Moment and Vibrational Modes of the Centrosymmetric Indigo Molecule. The J Phy Chem A (2020) 124:10856-10869. doi:10.1021/acs.jpca.0c09791 
58. Gilman Y, Allen PB, and Hybertsen MS. Density-functional Study of Adsorption of Isocyanides on a Gold (111) Surface. J Phys Chem C (2008) 112:3314-20. doi:10.1021/jp0745541

59. Kiguchi M, Miura S, Hara K, Sawamura M, and Murakoshi K. Conductance of a Single Molecule Anchored by an Isocyanide Substituent to Gold Electrodes. Appl Phys Lett (2006) 89:213104. doi:10.1063/1.2392816

60. Xue Y, Li X, Li H, and Zhang W. Quantifying Thiol-Gold Interactions towards the Efficient Strength Control. Nat Commun (2014) 5:4348-9. doi:10.1038/ ncomms 5348

61. Hong W, Manrique DZ, Moreno-García P, Gulcur M, Mishchenko A, Lambert CJ, et al.Single Molecular Conductance of Tolanes: Experimental and Theoretical Study on the junction Evolution Dependent on the Anchoring Group. J Am Chem Soc (2012) 134:2292-304. doi:10.1021/ja209844r

62. Widawsky JR, Chen W, Vázquez H, Kim T, Breslow R, Hybertsen MS, et al.Length-Dependent Thermopower of Highly Conducting Au-C Bonded Single Molecule Junctions. Nano Lett (2013) 13:2889-94. doi:10.1021/nl4012276

63. Park S, Kang H, and Yoon HJ. Structure-thermopower Relationships in Molecular Thermoelectrics. J Mater Chem A (2019) 7:14419-46. doi:10.1039/ c9ta03358k

64. Madadi Asl M, Vahabie AH, and Valizadeh A. Dopaminergic Modulation of Synaptic Plasticity, its Role in Neuropsychiatric Disorders, and its Computational Modeling. Basic Clin Neurosci (2019) 10:1-12. doi:10.32598/ bcn.9.10.125

65. Sun J, Fu Y, and Wan Q. Organic Synaptic Devices for Neuromorphic Systems. J Phys D Appl Phys (2018) 51:314004. doi:10.1088/1361-6463/aacd99

66. Prasad N, Pramanik T, Banerjee SK, and Register LF. Realizing Both Shortand Long-Term Memory within a Single Magnetic Tunnel junction Based Synapse. J Appl Phys (2020) 127:093904. doi:10.1063/1.5142418

67. Madadi Asl M, Valizadeh A, and Tass PA. Dendritic and Axonal Propagation Delays Determine Emergent Structures of Neuronal Networks with Plastic Synapses. Scientific Rep (2017) 7:1-12. doi:10.1038/srep39682
68. Madadi Asl M, Valizadeh A, and Tass PA. Delay-induced Multistability and Loop Formation in Neuronal Networks with Spike-timing-dependent Plasticity. Sci Rep (2018) 8:12068-15. doi:10.1038/s41598-018-30565-9

69. Nandakumar SR, Le Gallo M, Boybat I, Rajendran B, Sebastian A, and Eleftheriou E. A Phase-Change Memory Model for Neuromorphic Computing. J Appl Phys (2018) 124:152135. doi:10.1063/1.5042408

70. Madadi Asl M, Valizadeh A, and Tass PA. Propagation Delays Determine Neuronal Activity and Synaptic Connectivity Patterns Emerging in Plastic Neuronal Networks. Chaos (2018) 28:106308. doi:10.1063/1.5037309

71. Madadi Asl M, Valizadeh A, and Tass PA. Dendritic and Axonal Propagation Delays May Shape Neuronal Networks with Plastic Synapses. Front Physiol (2018) 9:1-8. doi:10.3389/fphys.2018.01849

Conflict of Interest: The authors declare that the research was conducted in the absence of any commercial or financial relationships that could be construed as a potential conflict of interest.

Publisher's Note: All claims expressed in this article are solely those of the authors and do not necessarily represent those of their affiliated organizations, or those of the publisher, the editors and the reviewers. Any product that may be evaluated in this article, or claim that may be made by its manufacturer, is not guaranteed or endorsed by the publisher.

Copyright (c) 2021 Ramezani Akbarabadi and Madadi Asl. This is an open-access article distributed under the terms of the Creative Commons Attribution License (CC $B Y)$. The use, distribution or reproduction in other forums is permitted, provided the original author(s) and the copyright owner(s) are credited and that the original publication in this journal is cited, in accordance with accepted academic practice. No use, distribution or reproduction is permitted which does not comply with these terms. 